\title{
Psychiatry with Teeth: Notes on Coercion and Control in France and the United States
}

\author{
Anne M. Lovell • Lorna A. Rhodes
}

Published online: 14 November 2014

(C) Springer Science+Business Media New York 2014

\begin{abstract}
"Psychiatry with teeth": with just three words, a head psychiatrist in Lyon, France, captures the confined spaces and internal discipline of a new type of intensive treatment center for "difficult patients" (Velpry and Eyraud 2014). His pithy phrase provides a vivid yet disconcerting image for the 'rough edge' of psychiatry where, as the editors of this issue of Culture, Medicine, and Psychiatry devoted to emergent forms of care and control remind us, constraint and coercion signal a fundamental division between patients and clinicians ${ }^{1}$ (Velpry and Brodwin 2014).

At the level of institutions and the state, it is through constraint that the larger social "will" (to power, to social order) shows its teeth. We can understand "teeth" at the material level as the community supervision, restraints, locked units, quiet rooms, involuntary hospitalizations, and (in some cases) medications through which the will of clinicians and the power of institutions are enacted upon the bodies of psychiatrically defined individuals. Each of these papers offers a close reading of some aspect of this enactment at the local level. In the relationships constitutive of psychiatric practice, constraint and coercion mark the sites of greatest conflict between patient, clinician, and institution - the places where teeth are most likely to be bared in disputes over the moral status of the patient and the legitimacy of the institution. 'Teething pains' afflict those involved in this dynamic, pains of growth as individual clinicians learn to accept constraint as a necessary aspect of their work.
\end{abstract}

\footnotetext{
${ }^{1}$ At the session of the meetings of the American Anthropological Association where earlier versions of these papers were presented, David Oaks, a leader in the movement for human rights of former and current psychiatric patients (or "survivors"), presented the patient's view of this relationship.
}

\footnotetext{
A. M. Lovell ( ()

Inserm Cermes3, Université Paris Descartes, Paris, France

e-mail: anne.lovell@parisdescartes.fr

L. A. Rhodes

Department of Anthropology, University of Washington, Seattle, USA

e-mail: 1rhodes@u.washington.edu
} 
For mental health workers in training, like Henckes' nurses in a pilot social psychiatry program, or for those facing new situations, like Brodwin's psychiatrist caught between institutional contradictions in a resource-poor environment, the practice of constraint rubs up against their professional and ethical sense of themselves (Brodwin 2014; Henckes 2014). Professional socialization involves their own "teething"- the painful acquisition of constraint techniques, through forms of self-reflection-which is precisely what Hejtmanek's front-line workers at an adolescent psychiatric custody residence (and some of Henckes's nurses) are rejecting (Hejtmanek 2014). On the contrary, the psychiatrists facing the expansion of hybrid forms of confinement (part elements of the asylum, part components of the prison) struggle to elaborate a discourse of constraint as a therapeutic necessity rather than as a form of socially mandated control or "social defense" (Velpry and Eyraud 2014).

As the editors announce in their introduction, the twin imperatives of care and confinement, cure and custody, have always defined psychiatry. Why then focus particularly on France and the United States? And what, precisely, is emergent today?

\section{Trans-Atlantic Legacies}

In the second half of the twentieth century, scholars in France and the United States independently developed a theoretical and empirical body of scholarship necessary to the anthropological understanding of care and constraint. This corpus included the works of Michel Foucault, Robert Castel, David Rothman and other historians, Gladys Swain, Marcel Gauchet and Erving Goffman as well as a number of early NIMH-funded anthropologists and sociologists. Of particular interest for this issue of Culture, Medicine, and Psychiatry is the observation that French scholars of that era tended to highlight "the incarcerative practices of psychiatry as epitomized in the asylum" (p. 6), a perspective which historian Jan Goldstein suggests-in the case of Foucault, Castel, Gauchet, and Swain-stemmed more from the authors' (leftist) concerns with issues of their own time than from the directions suggested by the archives themselves (Goldstein 1987), pp. 3-6. Regardless of whether one accepts Goldstein's critique, we can discern a liberal (in the American sense) counterpart to the interest French scholars shared for the origins and purposes of psychiatric confinement, in the social control theories illustrated by Goffman's classic study of the asylum and by the symbolic interactionism studies of his contemporaries. In the following years, a proliferation of ethnographies of psychiatry in the United States, and the incipient sociology of psychiatry in France turned to other questions. Nevertheless, the themes of constraint, confinement, and social control persist as a major heritage of these two nationally anchored sets of perspective and problematic. The English bias of international academia and publishing (and our own limitations), of course, precludes identifying a similar heritage in sociologies and anthropologies in other countries. ${ }^{2}$ However, the French

\footnotetext{
${ }^{2}$ Klaus Doerner's classic Marxist perspective on confinement and social control provides a counterexample of a widely translated study read by scholars of psychiatry in Europe (Doerner and Klaus 1969/ 1977). Studies of psychiatry influenced by the early Foucault also appeared in the UK and Canada.
} 
and US perspectives have greatly influenced research elsewhere, concerning, for example, contemporary forms of governmentality and the organization and ideological bases of colonial and post-colonial psychiatry.

Beyond parallel legacies, a second affinity underlies the juxtaposition of this set of papers. French and American scholars and public intellectuals represent a sort of alterity, each for the other, particularly with regard to ideas about "humanism," "freedom," "the subject," "autonomy"-keywords that run through the papers-and "health democracy" (especially in France), "citizenship," "human rights," and related concepts. The tension between these key concepts and actual practices of constraint and care in much of the contemporary "West" traverses not only public debate to differing degrees but also the everyday work of mental health professionals, as the papers show.

While the papers do not allow reasoned comparisons of the extent to which practices of constraint are multiplying in each country — or even of the range of forms they takethey do present careful readings of what might be elements of the different assemblages from which constraint and care emerge today. At a pragmatic level, comparisons between these landscapes are impeded by the very different organization of psychiatry in France versus the United States. In France, a centralized state and national laws establish a relative coherence across, or similarity among structures of care and constraint at local levels, although implementation of national frameworks is uneven and regionalization allows for some local initiatives. Model and experimental programs-such as the original UMD ("unit for difficult patients") established in 1910 in Villejuif, outside Paris; and the experimental training program for psychiatric nurses, in the 13th arrondissement of Paris - also break the homogeneity of institutionalized care and constraint (Lovell 2010). In the United States, despite clear tendencies in mental health care and constraint across the 50 states, state legislation, organizational structures, and modes of constraint and care differ far more between localities, states, and regions than across French territories. The relationship between American psychiatry and criminal justice (Rhodes 2004) and the proliferation of private-public partnerships and privatization in those fields also suggest a different configuration than in France where, to cite but one example, the Ministry of Health is responsible for the health care of prisoners. We should also heed against simple nominalism-assuming a correspondence between elements referred to by the same abstract term (for example, "care" or "outpatient commitment" or "isolation room" or "dangerousness") in different contexts. With these papers, we might ask what specific effects are produced by similar and dissimilar techniques, values, affects, conceptual practices and meanings and the particular assemblages to which they are attached.

\section{The Emergent}

Despite the above limitations, the papers succeed in providing elements of an eventual cartography of constraint and control practices, as they are emerging in France and in the U.S. What is "emergent" today, to echo the editors' term, follows the traces left from the post-asylum era, a historical period in which psychiatric institutions of confinement did not disappear so much as they were reconfigured and their forms of power multiplied along capillary lines that joined the hospital with 
other institutional apparatuses (dispositifs) beyond it (Rhodes 1991). Alongside these residues, psychiatric constraint has appeared at new sites: within communitybased psychiatry (e.g., outpatient commitment), through trans-institutional or interagency collusion and collaboration (e.g., court-ordered release to psychiatric care in lieu of prison), and as a trans-institutionalization of patients (e.g., the rising proportion of people with mental illness in prisons) as well as of psychiatric services (e.g., separate treatment units for prisoners with mental illness).

But constraint also emerges at the level of the individual clinician or front-line mental health worker through the process of learning to exert control and enact constraint. It is a particularity shared by these papers-a methodological point of entry to constraint through the mental health professionals themselves-that allows us to grasp that level. Henckes' and Hejtmanek's papers, in particular, illustrate that professional socialization process very well. The "apprenticeship in constraint", as Henckes calls it, also involves "the constraint of apprenticeship." The "new psychiatric nurse" in the model program of the post-war sectorisation (territoriallybased psychiatry) must reveal and reflect on his or her shortcomings and emotions, through written and oral procedures reminiscent of both confession and psychotherapy. Similarly, Hejtmanek's staff members, already well schooled in how to work with troubled youth in "psychiatric custody," are suddenly asked to reflect on their own emotional reactions to the consequences of applying constraintconsequences that include further violence on the part of the adolescent patients they attempt to bring under control. Psychoanalysis, a "philosophy of liberty," shapes this professional socialization through the emphasis on transference and counter-transference (in Hejtmanek's example) and self-analysis (for Henckes' nurses). In both papers, the coercive relationship between supervisor (or trainer) and mental health worker mirrors, albeit with less far-reaching consequences, the coercion those workers come to exercise upon their own patients.

\section{Conclusion: Always Teeth?}

From an anthropological viewpoint, not only can there not be a psychiatry without teeth; there can be no freedom without the limits set through relationality. As one French psychiatrist comments about the new mental health units for prisoners, "A secured and closed unit: this is what allows for all the other units to be open" (Velpry and Eyraud 2014). Limits may, of course, be expressed as pre-existing norms and structures. More fundamentally, limits allow sociality, through a mutual or coeval process, to come into being in the first place; they constantly re-emerge as a necessary condition for the on-going co-existence (under whatever forms) of empirical, acting, and interacting individuals - individuals in flesh-and-blood rather the ideal abstraction of free-standing "individuals." This principle of relationality undergirds even the most tolerant of mental health structures, such as those organized along the principles of patient "choice" or "autonomy" or "clientcenteredness" (Lovell and Cohn 1998).

By exploring the shape of this co-existence in the small details of daily clinical and inter-institution interactions, these papers remind us not only that psychiatry has 
teeth, but that teeth fix the limits emerging from a variety of conditions of possibility. By offering a window onto the recurring uneasiness of those charged with enforcing those limits, each author has revealed the highly instable, problematic nature of that enforcement. Perhaps, new windows can be opened onto the fraught nature of the therapeutic project, while allowing anthropologist to better understand why modes of constraint are constantly re-invented, even in new contexts.

\section{References}

Brodwin, Paul

2014 The Ethics of Ambivalence and the Practice of Constraint in US Psychiatry. Culture, Medicine, and Psychiatry. doi:10.1007/s11013-014-9401-z.

Doerner, Klaus

1969/1977 Madmen and the Bourgeoisie. New York: Urizen Books.

Goldstein, Jan E.

1987 Console and Classify: the French Psychiatric Profession in the Nineteenth Century. Cambridge: University of Chicago Press.

Hejtmanek, Katie R.

2014 Practicing and Resisting Constraint: Ethnography of "Counter Response" in American Adolescent Psychiatric Custody. Culture, Medicine, and Psychiatry. doi:10.1007/s11013-0149403-x.

Henckes, Nicolas

2014 Learning Constraint. Exploring Nurses' Narratives of Psychiatric Work in the Early Years of French Community Psychiatry. Culture, Medicine, and Psychiatry. doi:10.1007/s11013-0149399-2.

Lovell, Anne M.

2010 Santé Mentale. In Santé Publique. L'état des Savoirs. D. Fassin and B. Hauray, eds., pp. 161162. Paris: La Découverte.

Lovell, Anne M., and Sandra Cohn

1998 The Elaboration of "Choice" in a Program for Homeless Persons Labeled Psychiatrically Disabled. Human Organization 57(1): 8-20.

Rhodes, Lorna A.

1991 Emptying Beds: the Work of an Emergency Psychiatric Unit. Berkeley: University of California Press.

Rhodes, Lorna Amarasingham

2004 Total Confinement: Madness and Reason in the Maximum Security Prison. Berkeley: University of California Press.

Velpry, Livia, and Paul Brodwin

2014 The Practice of Constraint in Psychiatry: Emergent Forms of Care and Control. Culture, Medicine, and Psychiatry. doi:10.1007/s11013-014-9402-y.

Velpry, Livia, and Benoît Eyraud

2014 Confinement and Psychiatric Care: A Comparison Between High-Security Units for Prisoners and for Difficult Patients in France. Culture, Medicine, and Psychiatry. doi:10.1007/s1 1013-014-9400-0. 